\title{
Luces y sombras de la política de educación intercultural bilingüe en Honduras
}

Ricardo Morales Ulloa, António M. Magalhães

Faculdade de Psicologia e de Ciências da Educação da Universidade do Porto, Portugal, moralesu@yahoo.com,antonio@fpce.up.pt

\section{Resumen}

Tomando en cuenta la gran diversidad de lenguas y culturas en América Latina, con propósitos de cristianización y de hispanización durante la colonia española se utilizaron medidas como la utilización de lenguas gestuales, el uso de intérpretes de linguas francas (nahuatl y quechua) y la enseñanza religiosa en lenguas vernáculas, consideradas "inferiores" pero útiles para tal propósite. Posteriormente, las independencias latinoamericanas, en su afán de construir los estados nacionales unitarios establecieron políticas que solamente reconocían la lengua castellana y consideraron que las culturas originarias, diferentes del imaginario nacional blancomestizo, eran obstáculos para el desarrollo del Estado moderno. De esta manera, más allá de su secular discriminación y marginalidad, los pueblos culturalmente diferenciados fueron oficialmente invisibilizados hasta los años 1960, cuando surge el reclamo por una ciudadanía basada en los derechos culturales, este hito marca el inicio de los programas de educación bilingüe- intercultural. Esta comunicación analiza las características de esta política en Honduras, sus luces y sus sombras y los retos que tiene aún por delante para conformar la nación hondureña diversa e incluyente.

Palabras clave: educación, interculturalidad, bilingüismo, pueblos culturalmente diferenciados. 


\section{Abstract}

Given the great diversity of languages and cultures in Latin America the Spanish colonialists, for purposes of Christianization and Hispanization, took different measures as the use of sign language, the use of interpreters from and to lingua franca (Nahuatl and Quechua) and the religious teaching in vernacular languages, considered as "inferior and wild" but fit for purpose. Subsequently, the processes of Latin American independence, in an effort to build the nation states unity have established policies on the basis of the official recognition of the Spanish language assuming that the original cultures, different from the national imaginary white-mestizo, were an obstacle to the development of the modern state. Thus, beyond its secular discrimination and marginalization, culturally differentiated people were made officially invisible until the 1960's. By that time, there was a movement claiming for citizenship based on cultural rights, this milestone marks the beginning of the bilingual-education programs exchange. This paper analyzes the characteristics of these policies in Honduras, the lights and shadows that it spreads over the Honduran context that is dealing with major challenges in the construction of a diverse and inclusive nation.

Key words: education, interculturallity, bilingualism, culturally differentiated people

\section{Introducción: La diversidad cultural de América Latina y Honduras}

Históricamente, América Latina ha sido un mosaico de lenguas y culturas. Son ampliamente conocidas las grandes civilizaciones maya y azteca que se desarrollaron en la región meso-americana, ubicada geográficamente en lo que actualmente son los territorios de México y Centroamérica. La civilización maya, extinta antes de la conquista española, sorprende a los investigadores especialmente por el avanzado conocimiento en matemáticas y astronomía, de hecho en la actualidad en el mundo occidental se realiza una discusión, trivial 
a mi criterio, sobre el fin del mundo a partir de las interpretaciones del calendario maya; los aztecas, por su parte a la llegada de los conquistadores constituían un imperio sólidamente establecido en el cual se hablaba el nahualt, una lengua muy influyente aún en la actualidad'; en América del Sur, los incas ${ }^{2}$ también conformaban un imperio y de hecho con los aztecas constituían la concentración de población más grande de la América precolombina. Lo cierto es que además de estas tres grandes culturas³, a la llegada de los conquistadores españoles, existían una gran cantidad de otros pueblos y lenguas ${ }^{4}$ que conformaban una enorme mosaico lingüístico y cultural (Herranz, 2008).

A medida que la colonización se expande los españoles se percatan de la gran cantidad de lenguas y culturas diferentes, el caso de Honduras es muy ilustrador, en este sentido, Herranz, citando una carta escrita por un Frayle al Rey Felipe II de España, dice lo siguiente: “... y en cada una de las provincias hay y hablan los indios diferentes lenguas, que parece que fue el artificio más mañoso que el demonio tuvo en estas partes para plantar discordia, confundiéndolos con tantas y tan diferentes lenguas que tienen como son..." (2008: 50) y enumera las lenguas. Evidentemente, esta auténtica Torre de Babel, es una enorme dificultad para la cristianización y castellanización pretendida por la corona española, de tal manera que para resolver este problema se adoptan diversos medios, por ejemplo: el uso de lenguas de señales como en México donde los españoles crearon una escuela de "mímica" técnica teatral usada por los aztecas, la utilización de traductores indígenas que aprendían el castellano y dominaban lenguas originarias

\footnotetext{
1 Palabras como tomate o chocolate son de origen nahualt, de hecho estos son dos de los aportes más importantes de México a la culinaria europea. El nahualt es una de las lenguas amerindias que más ha influido al castellano que se habla en la actualidad.

${ }^{2}$ El quechua era la lengua dominante en el imperio inca, en la actualidad junto con el aymara son las lenguas originarias que tienen el mayor número de hablantes en América del Sur. El Aymara es la lengua materna del Presidente boliviano Evo Morales.

${ }^{3}$ El colapso de la civilización maya, ocurrido antes de la conquista española, no significó el desaparecimiento de los pueblos y culturas mayas. De manera ligera se habla a veces del desaparecimiento de los mayas.

${ }^{4}$ Como ejemplo el grupo de lenguas tupí guaraní a la cual pertenecen el tupinamba que ha influido al portugués hablado en Brasil y el guaraní la lengua nacional y oficial de Paraguay.
} 
hegemónicas y difundidas como linguas francas en diferentes regiones (el nahuatl en México y el quechua en el Perú)5; en algún momento, también los frailes españoles asentados en cada pueblo indígena, aprendieron lenguas originarias, para utilizarlas como puente hacia el castellano. Finalmente se impuso la política lingüística de "sólo castellano" y con ésta la destrucción sistemática de las lenguas originarias consideradas de todas maneras salvajes e inferiores (Idem, 2008).

Este propósito casi se logró, pero algunas de las lenguas y culturas lograron sobrevivir gracias a las más diversas maneras de resistencia tales como el uso de la lengua materna en situaciones familiares, aún y cuando se prohibía; el aislamiento en grandes barreras naturales como las selvas centroamericanas, la amazonia y las altas montañas de los Andes también facilitó la sobrevivencia de algunos pueblos originarios (Arciniegas, 1989). Superada la colonización española, a inicios del siglo XIX las independencias de los países latinoamericanos ${ }^{6} y$ el afán por la construcción de los estados unitarios marcan otra etapa de asimilacióna la ya bien consolidada cultura hegemónica blanco-mestiza producto directo a la herencia hispánica (Torres Rivas, 1995). De paso sea dicho, las ideas ilustradas europeas que inspiraron las independencias y consecuentemente las constituciones políticas de los Estados latinoamericanos, afirmaron también una noción de modernidad que consideraba a las culturas no europeas como atrasadas y supersticiosas.

En su idea de conformar estados nacionales unitarios, sobre la base de una identidad cultural blanco-mestiza común, las constituciones latinoamericanas asumieron el español y el portugués en el caso de

\footnotetext{
${ }^{5}$ El Inca Garcilaso de la Vega, cronista extraordinario de la conquista del Perú, cuenta como Felipe, traductor originario, traduce a Atahualpa, último emperador inca, el dogma de la trinidad cristiana "Dios trino y uno" es traducido como "Dios tres más uno son cuatro", parece entonces que Atahualpa, ante la irritación de los conquistadores, insistía en que los blancos tenían cuatro dioses. Desde la cosmovisión panteísta esta idea de un Dios único resulta incomprensible (SilvaSantisteban, 2010).

${ }^{6}$ Con la excepción de Haití que logró su independencia en 1804 mediante una revuelta de esclavos, ningún movimiento de independencia en América Latina fue producto del levantamiento de los pueblos originarios.
} 
Brasil como lenguas nacionales-oficiales y los sistemas educativos se convirtieron en medios privilegiados para lograr este propósito; de esta manera, el proyecto uniformador tornó invisibles a los pueblos culturalmente diferenciados y los excluyó de participar activamente en la vida social, económica y política de los países. Sin embargo, en la actualidad sobreviven unos 40 millones de indígenas que equivalen al $10 \%$ de la población latinoamericana y en algunas países como Bolivia y Guatemala7 constituyen la mayoría de la población, adicionalmente se hablan unas 500 lenguas indígenas (López y Küper 2001). En el caso de Honduras la población está conformada mayoritariamente por mestizos, pero existen seis pueblos indígenas originarios y dos pueblos negros de origen africano, con lo cual, siguiendo la tendencia general de América Latina por lo menos un $10 \%$ de la población hondureña es culturalmente diferenciada (Amaya, 2011).

\section{Interculturalidad y educación intercultural: una mirada desde América Latina}

La idea de interculturalidad en América Latina ha sido desarrollada en el contexto del movimiento indígena que se inicia en los años 1970 y se fortalece a partir de la transición democrática experimentada en los años 1980; de tal manera, que es parte de los reclamos relacionados con la opresión y la exclusión histórica de los pueblos culturalmente diferenciados. Junto con esta demanda implícita de justicia social, basada en la participación económicapolítica y el reconocimiento cultural, la interculturalidad como lo afirma López "es una propuesta de diálogo, intercambio y complementariedad que apunta hacia la articulación de las diferencias pero no a su desaparición bajo el lema de la unidad en la diversidad" (2001:9). En síntesis, la interculturalidad no es apenas “justicia social para los indios" sino fundamentalmente el reconocimiento y la convivencia armónica de toda la diversidad étnica, lingüística y cultural que caracteriza a las sociedades

\footnotetext{
${ }^{7}$ El $60 \%$ de la población de Guatemala es indígena y se hablan 22 lenguas de la familia lingüística maya (Moya, 1999)
} 
latinoamericanas. Naturalmente también, este concepto como sus similares europeos es una respuesta al racismo, la xenofobia y a todas las formas de discriminación.

La exclusión antes referida es una de las causas determinantes de la pobreza que aqueja a los pueblos indígenas en América Latina, tal es así que se considera que ser indígena equivale a ser pobre y más aún ser indígena y mujer es una condición doblemente vulnerable (Anderson, 2004). Como es sabido, la desventaja socioeconómica tiene impactos negativos en la educación; en este sentido, los sistemas educativos latinoamericanos han fallado al no garantizar el derecho a la educación de su población, especialmente indígena, y segundo al ignorar desde las políticas educativas las condiciones pedagógicas particulares que demandan los procesos educativos en contextos culturales y lingüísticos diferenciados. Como resultado de estas formas de exclusión, en el caso de Honduras, las regiones con mayor concentración de población indígena y afro-descendiente presentan los índices más altos de analfabetismo y rezago educativo del país (PNUD, 2010; Alas y Moncada, 2009).

La lucha histórica de los pueblos indígenas en América Latina ha sido también por el derecho a la educación. En una primera etapa la prioridad se fijaba en la alfabetización en la lengua hegemónica, evidentemente porque ésta era el punto de partida para defender los derechos de los pueblos originarios en los espacios políticos establecidos por la dominancia blanco-mestiza de la nación homogénea ${ }^{8}$. Posteriormente en los años 1960 y 1970 los gobiernos, como en la colonia, recurrieron al uso provisorio de las lenguas originarias para facilitar la apropiación de la lengua hegémonica, en el llamado bilingüismo de transición, no obstante el curriculum nacional continuaba ignorando la diferencia cultural. En la década 1970 con el fortalecimiento del movimiento indígena se introduce un nuevo enfoque de educación bilingüe que fomenta el desarrollo

\footnotetext{
${ }^{8}$ Los miskitu de la región oriental de Honduras no son bilingües, para este pueblo el español es segunda lengua, desde su experiencia docente, el autor de este texto conoce las dificultades que los estudiantes de esta cultura enfrentan en las aulas universitarias, debido al desconocimiento del español la lengua oficial del país.
} 
de la lengua materna y de la segunda lengua y partiendo de esta experiencia desde los años 1980 comienza a modificarse el curriculum en la vía de considerar las cosmovisiones tradicionales, de esta manera de la educación bilingüe se realiza la transición a la educación intercultural bilingüe (López, 2001).

Para López 2001 la educación intercultural bilingüe es una educación que tiene como referente inmediato la cultura propia de los educandos pero que está abierta a la incorporación conocimientos y saberes de otras culturas, incluida la cultura universal, en el campo lingüístico propiamente, es movilizada en una lengua amerindia y en castellano o portugués, lo cual fortalece las competencias comunicativas en la lengua materna y en una segunda lengua. La educación intercultural bilingüe no es apenas educación para indígenas o enseñanza en lenguas indígenas, es una concepción de educación que pretende desarrollar actitudes y acciones positivas con respecto a la diversidad, que es parte de la vida cotidiana de las sociedades latinoamericanas, por esta razón la educación intercultural bilingüe pretende ser una propuesta curricular transversal del curriculum para la formación de las sociedades, de manera tal, que éstas sean capaces de convivir armónicamente asumiéndose diversas.

\section{El reconocimiento de la nación culturalmente diversa en Honduras}

A lo largo del período post-colonial la diversidad cultural y lingüística de Honduras ha sido invisible. La afirmación general es que somos un país homogéneo, compuesto mayoritariamente por mestizos y en consecuencia el aparato político del país respeta esa condición y la fija como norma que define el perfil de la hondureñidad. En tal sentido, todas las constituciones emitidas después de la colonia declararon al español como lengua oficial y única del país, es más, en la constitución de 1982 vigente en la actualidad, los legisladores fueron más allá al afirmar que es deber del Estado proteger la pureza de "nuestra lengua" la lengua española e incrementar su enseñanza”. A partir de este mandato constitucional el sistema 
educativo hondureño ha reconocido al español como lengua única de instrucción y con ella los valores dominantes de la cultura blancomestiza heredada de la colonia.

La ignorancia y el ocultamiento de las lenguas de los pueblos culturalmente diferenciados era tan marcada en Honduras, que hasta los años 1970, los espacios curriculares dedicados a la lengua española eran denominados como "idioma nacional", en la misma década una reforma educativa enmendó este error fundamental, porque de hecho el castellano puede ser la lengua oficial de Honduras pero por su origen no es una lengua nacional; de todas maneras, corregir el error de forma no alteró la sustancia y el sistema educativo continuaba siendo fundamentalmente españolizante. La alfabetización de los pueblos indígenas se ha realizado en español y con maestros hispanohablantes, en este contexto, la escuela es el medio privilegiado para afirmar la identidad nacional, por lo tanto se ha creído que esta política no tiene más que la buena intención de "hacer hondureños a los indios". Amaya considera que esta "política de exclusión lingüística" (2011:147) ha sido en parte causal de la reciente extinción de algunas lenguas sobrevivientes de la colonia como el lenca y el maya-chortí.

Haciendo eco del movimiento indígena que se extiende por América Latina, en los años 1970, los pueblos indígenas y afro-caribes de Honduras se organizan en primera instancia para reclamar el derecho a mantener y recuperar sus tierras ancestrales de las cuales habían sido gradualmente despojados en los últimos 200 años (Herranz, 1995). A finales de los años 1980 el reclamo por la tierra se extiende a la defensa de cultura y la lengua. En el año 1992 con motivo de los quinientos años del descubrimiento de América, junto con las declaraciones oficiales que hacen apología del "encuentro de dos mundos", el movimiento indígena exige una relectura histórica de la conquista y llama la atención sobre sus condiciones actuales de exclusión social, económica y política. Como consecuencia de estas luchas, en 1994, el gobierno de Honduras aprueba el Acuerdo Presidencial 0719-EP con el cual el Estado reconoce "el carácter "pluricultural y plurilingüe” de la sociedad hondureña. 


\section{Las luces y las sombras}

Si bien Honduras no realizó reformas a su constitución para reconocer su carácter de nación culturalmente diversa, el Acuerdo 719 marca un giro radical en dos sentidos: en la política lingüística al reconocer el plurilingüismo y en la política educativa al establecer la educación intercultural bilingüe. El primer considerando del acuerdo 719 expresa "Honduras es un país pluricultural y multiétnico que requiere institucionalizar la educación bilingüe intercultural para responder a la riqueza y diversidad cultural", en adelante el cuarto considerando, reconoce las fallas del Estado y su sistema educativo al no reconocer la diversidad cultural " el sistema educativo nacional ha adolecido de una política definida para la educación bilingüe intercultural, con una currícula diferenciada para la conservación de los diversos componentes y expresiones de la cultura nacional". La luz histórica de este acuerdo radica en que como afirma Amaya el Estado hondureño "reimagina la naturaleza y la esencia de la nación en términos de una sociedad multicultural y pluriétnica" (2011: 147) sin duda alguna un paso importante en la creación de una sociedad más justa e incluyente.

Como suele suceder en Honduras entre las declaraciones y la realidad existen notables diferencias. Herranz (1995) destaca la pertinencia del concepto de educación intercultural bilingüe presente en el acuerdo 719, sin embargo, se requiere reflexionar sobre los obstáculos que enfrenta una visión educativa plural y avanzada como ésta en una sociedad tan desigual como la hondureña. De hecho asumirnos como diversos y a la vez como hondureños todos y todas requiere de construir el imaginario de la nación homogénea. La sociedad hondureña continúa pensando que al no existir conflictos étnicos evidentes, tampoco somos racistas y consecuentemente la discusión sobre la diversidad cultural no es algo que tenga mucho sentido, no obstante, las alusiones a indios y a negros continúan siendo peyorativas 9 y la distribución de las oportunidades todavía tiene matices racistas, así lo expresa un

\footnotetext{
${ }^{9}$ En Honduras es común escuchar bromas de contenido racista como "se me salió el indio" para referirse a comportamientos impulsivos e irracionales.
} 
funcionario del Programa Nacional de Educación de las Etnias Autóctonas de Honduras (PRONEEAH) "Tenemos Ministros de Cultura y Deportes negros, pero aún no tenemos un Ministro de Finanzas, ni de Educación y menos un Presidente de la República".

En el caso de Honduras es necesario profundizar el análisis de la educación intercultural bilingüe (EIB) en dos dimensiones: la social y la pedagógica, la primera, bajo una visión de equidad, implica que el modelo educativo intercultural es para toda la sociedad hondureña y no apenas para los pueblos culturalmente diferenciados de la mayoría mestiza y la segunda ligada a la primera es el camino por el cual este modelo educativo trasciende el nivel retórico y se convierte en práctica. Como hasta ahora la EIB se ha asociado más al bilingüismo, está socialmente asumido que siendo monolingüe la mayoría mestiza la EIB no es de interés general, sino más bien "educación para indios y negros que viven en el campo" de hecho la EIB tiene estas dos características, es un programa especial del Ministerio de Educación para los pueblos culturalmente diferenciados y se aplica principalmente en comunidades rurales. De manera contrastante, existe en Honduras un modelo de educación bilingüe, privado, en lenguas europeas, especialmente inglés, desarrollado antes que la EIB y que tiene mucho prestigio social por ser la educación por excelencia de las clases altas.

En lo que refiere a la dimensión pedagógica, conceptualmente la EIB ha sido principalmente creación de antropólogos y lingüistas y en menor grado de pedagogos, de esta situación se desprende la dificultad de convertir concepciones abstractas en diseños curriculares, formación de maestros o materiales didácticos adecuados para llevar los conceptos de la EIB a la práctica en aula de clase. En Honduras si bien el curriculum nacional para la educación básica considera a la interculturalidad como un eje transversal, a pocos queda claro que elementos de las culturas se deben tratar en la escuela y con qué recursos metodológicos, en el mejor de los casos, los profesores al no tener la formación necesaria en ElB hacen sus propias interpretaciones, con frecuencia desde la mirada 
folklórica, que a la larga refuerza las ideas estereotipadas que la cultura dominante tiene sobre los pueblos culturalmente diferenciados. Consecuentemente, hace falta permear la formación docente con un concepto pertinente de interculturalidad y de la misma manera poner a la disposición de los maestros los materiales didácticos necesarios para movilizar tal concepto.

El bilingüismo pese a ser la experiencia educativa con referentes culturales más antigua en Honduras, también tiene sus sombras. La primera dificultad que enfrenta es la escritura, porque no todas las lenguas de los pueblos culturalmente diferenciados se escriben y de hecho se han mantenido en gran parte como tradiciones orales. En este caso parece también que la realidad ha desbordado a las declaraciones, el acuerdo 719 había considerado que la EIB fomentaría un bilingüismo de mantenimiento y desarrollo de la lenguas originarias, movilizado por el uso de la lengua materna en todas áreas curriculares y el español como segunda lengua; evidentemente este enfoque demandaba la normalización de las lenguas originarias, una tarea que pese a sus avances aún tiene un camino largo por recorrer. Probablemente, este vacío ha determinado que el curriculum nacional no tenga definiciones pedagógicas puntuales sobre el bilingüismo y tampoco una selección de los contenidos que deben impartirse en cada lengua, lo cual aumenta el riesgo de que la EIB en la práctica no sea más que la traducción, frecuentemente imposible y culturalmente incompatible, del conocimiento occidental en la lengua hegemónica a las lenguas de los pueblos culturalmente diferenciados, que tienen escritura. 


\section{Consideraciones finales}

Habiendo avanzado en la creación de una institucionalidad favorable a los derechos lingüísticos y culturales (Moya, 1998), el compromiso con las políticas de educación intercultural bilingüe todavía parece depender más de coyunturas políticas y de los intereses particulares de los gobernantes de turno. A casi veinte años de haber reconocido formalmente la diversidad cultural y lingüística de la nación hondureña y declarado que la educación es el medio privilegiado para su mantenimiento y desarrollo, la EIB parece más un proyecto como otros y no un pilar del modelo educativo hondureño.

Una política más activa de EIB debería tener expresión en el presupuesto sectorial de educación y no depender casi exclusivamente de préstamos del Banco Mundial y de otros cooperantes internacionales. El Estado hondureño debe asumir que una declaración no basta y que la puesta en escena de la EIB requiere la movilización de una gran cantidad de recursos propios, que validen la esencia soberana de la EIB como aporte a la construcción de una sociedad más democrática e incluyente.

No obstante, Las luces proceden fundamentalmente de un movimiento social indígena y afro-hondureño vigoroso, que en los últimos veinte años ha fortalecido el reclamo orgánico de sus derechos. Pese a las sombras, paso a paso, los pueblos culturalmente diferenciados de Honduras avanzan, desde el reconocimiento de sus derechos culturales y lingüísticos a la "conquista" de los espacios sociales, económicos y políticos que históricamente les fueron negados. Desde el estigma y la vergüenza, los pueblos culturalmente diferenciados también han iniciado la recuperación de la estima perdida "en si mismos, en sus pueblos y en sus instituciones culturales” (López, 2001: 20), de nuevo hablar garífuna, pech, tawahka, tol, miskitu, sentirse lenca o maya, vuelve a ser motivo de orgullo, pero queda mucho por andar para restañar las heridas recibidas en quinientos años de historia. 


\section{Referencias bibliográficas}

Alas, Mario y Moncada, German (2009). Equidad y Calidad en la Educación Nacional. Problemas de equidad en el sistema educativo hondureño. Revista Iberoamericana de Evaluación Educativa, 3(3), 136-151. Recuperado en mayo o9 de 2011. http:// www.rinace.net/riie/numeros/vol3/-num3/art.pdf

Amaya, Jorge Alberto (2011). La educación intercultural bilingüe y la construcción de la nación pluriétnica en Honduras. En Ramón Salgado y Renán Rápalo (Coords.), Estado de la educación en Honduras (pp. 143-156). Tegucigalpa: Universidad Pedagógica Nacional Francisco Morazán.

Anderson, Jeanine (2004). Categorías de diferencia, trayectorias de desigualdad: Superar la pobreza femenina diversa en América Latina. En María Elena Valenzuela y Marta Rangel (Editoras), Desigualdades entrecruzadas: Pobreza, género, etnia y raza en América Latina (pp. 87-131). Santiago de Chile: Oficina Regional de la Organización Internacional del Trabajo para América Latina.

Arciniegas, Germán (1989) El continente de siete colores: historia de la cultura de América Latina. Bogotá: Santillana-Aguilar.

Herranz, Atanasio (2008). Estado, sociedad y lenguaje: la política lingüística en Honduras. Tegucigalpa: Guaymuras.

Herranz, Atanasio (1995). Política lingüística, interculturalidad y currículo: el caso de los lencas de Honduras. En Atanasio Herranz, Marvin Barahona y Ramón Rivas (editores), Educación bilingüe intercultural en Centroamérica y México (pp.154-207). Tegucigalpa: Guaymuras.

López, Luis Enrique (2001). La cuestión de la interculturalidad y la educación en América Latina. Santiago de Chile: UNESCO. Recuperado en octubre 25 de 2012. http://portal.oas.org/ 
LinkClick.aspx?fileticket=JUel5RfjUdE\%3D\&tabid=1652.

López, Luis Enrique y Küper, Wolfgang (1999). La educación intercultural bilingüe en América Latina: balance y perspectivas. Revista Iberoamericana de Educación, 20, 17-85.

Moya, Ruth (1999). Interculturalidad y Reforma Educativa en Guatemala. En Ruth Moya (Editora), Interculturalidad y educación: Diálogo para la democracia en América Latina (pp.1954). Quito: Ediciones Abya-Yala/Proyecto EBI Cooperación Técnica Alemana GTZ

Moya, Ruth (1998). Reformas educativas e interculturalidad en América Latina. Revista Iberoamericana de Educación, 17, 105187.

Programa de las Naciones Unidas para el Desarrollo PNUD(2010). Informe sobre el Desarrollo Humano Honduras 2008/2009: De la Exclusión Social a la Ciudadanía Juvenil. Tegucigalpa: PNUD.

Silva-Santisteban, Ricardo (2010). Garcilaso de la Vega traductor. Mutas Mutandis, 3(2), 235-248. Recuperado en noviembre 2 de 2012 de http://www.google.es/\#hl=pt-PT\&sclient=psy

Torres Rivas, Edelberto (1995). Consideraciones sobre la condición indígena en América Latina y los Derechos Humanos. San José de Costa Rica: Instituto Interamericano de Derechos Humanos. 